\title{
Elbow Torque May be Predictive of Anatomic Adaptations to the Elbow After a Season of Collegiate Pitching: A Dynamic Ultrasound Study
}

\author{
Lafi S. Khalil, M.D., Toufic R. Jildeh, M.D., Muhammad J. Abbas, B.S., \\ Chad L. Klochko, M.D., Courtney Scher, D.O., Marnix Van Holsbeeck, M.D., \\ Stephanie J. Muh, M.D., Eric C. Makhni, M.D., M.B.A., Vasilios Moutzouros, M.D., and \\ Kelechi R. Okoroha, M.D.
}

\begin{abstract}
Purpose: To determine whether elbow torque was associated with anatomic adaptations of the medial elbow following a season of competitive pitching. Methods: Pitchers from 3 collegiate baseball teams were recruited during the preseason for participation. Before the season, pitchers were recorded throwing 5 "game-speed" fastball pitches from a standard distance off a mound while wearing a wearable sensor baseball compression sleeve that calculates elbow torque, arm speed, arm slot, and arm rotation. Participants subsequently underwent dynamic ultrasound imaging of the medial elbow, including measurements of the ulnar collateral ligament (UCL) and ulnohumeral joint space to assess elbow laxity. Following a full season of competitive pitching, all testing was repeated, and statistical analysis comparing preseason to postseason sonographic findings was performed. Results: Twenty-eight collegiate pitchers underwent preseason sonographic and kinematic testing. Nineteen pitchers were available for postseason testing. The average age (standard deviation) and playing experience was 19.9 (1.2) and 14.7 (1.5) years. Compared with preseason, there were significant increases in postseason UCL thickness $(1.92 \pm 0.09$ vs $1.56 \pm 0.09 \mathrm{~mm}, P<.01)$ and elbow laxity $(1.77 \pm 0.23 \mathrm{vs} 1.15 \pm$ $0.22 \mathrm{~mm}, P=.028$ ) after a season of pitching. No significant changes in pitching kinematic measurements were observed between preseason and postseason testing. Preseason pitching kinematic measurements were significantly associated with increased UCL thickness (arm slot: beta estimate $-0.03 \pm 0.01, P=.011$ ) and reduction in elbow laxity (elbow torque: beta estimate $-0.03 \pm 0.01, P=.04$ ) after a season of pitching. Pitchers with increased body weight and arm length demonstrated reduced medial elbow torque during pitching $(P<.05)$. Conclusions: After a season of competitive pitching, adaptive changes of the medial elbow were demonstrated on dynamic ultrasound. However, the influence of pitching kinematic measurements on these adaptations are of small magnitude and unknown clinical significance. Although wearable sensor technology may have value in trending individual pitcher kinematics, no discrete threshold appears to predict the development of adaptive changes at the elbow. Level of Evidence: Level II, prospective observational study.
\end{abstract}

$\mathbf{T}$ hroughout the course of a collegiate season, pitchers place significant stress on their throwing arm, putting them at risk for elbow injuries and ulnar collateral ligament (UCL) tears. Studies have

From the Departments of Orthopedic Surgery (L.S.K., T.R.J., M.J.A., S.J.M., E.C.M., V.M.) and Musculoskeletal Radiology (C.L.K., C.S., M.V.H.), Henry Ford Hospital, Detroit, Michigan; and Department of Orthopaedic Surgery, Division of Sports Medicine, The Mayo Clinic, Rochester, Minnesota (K.R.O.), U.S.A.

The authors report the following potential conflicts of interest or sources of funding: S.J.M. reports other from Exactech, outside the submitted work. E.C.M. reports other from Stryker, Arthrex, Smith $\theta$ Nephew, Pinnacle, and Springer, outside the submitted work. V.M. reports other from Stryker, Arthrex, Smith $\theta$ Nephew, and Pinnacle, outside the submitted work. K.R.O. reports other from Stryker, Arthrex, Smith $\theta$ Nephew, and Zimmer, outside demonstrated that the peak torque in the medial elbow is experienced during the transition from the late cocking to early acceleration while throwing. The throwing motion also creates an opening valgus 
moment in the ulnohumeral joint. ${ }^{1-3}$ Traditionally, quantifying forces on the arm during the throwing motion relied on motion-capture video analysis. However, with the advent of wearable sensor technology, it has become possible to record elbow torque, among various other throwing parameters, in a simple and accessible manner. Boddy et al. $^{4}$ evaluated wearable sensors as an alternative to marker-based motion capture and found that measurements of arm slot, arm rotation, and elbow torque were significantly correlated with motion capture. Likewise, this device has been shown to measure elbow torque, arm speed, arm slot, and shoulder rotation with $95 \%$ precision $^{5-7}$ and serve as an accurate measure of medial elbow torque as compared with high-speed motion analysis $(r=0.93){ }^{8}$

It is understood that medial elbow torque during pitching is a risk factor for elbow injury. ${ }^{9}$ In addition, the repetitive stresses at the medial elbow from a full season of pitching may predispose to elbow injury in pitchers, related to attenuation of the static and dynamic restraints of the elbow. ${ }^{10-12}$ The physical properties of these restraints have been characterized in competitive baseball pitchers, with repetitive overhead throwing leading to downstream adaptive changes visualized on dynamic ultrasound, such as increased UCL thickness, UCL heterogeneity, and ulnohumeral joint space (UHJS) widening (i.e., elbow laxity). ${ }^{13-20}$ These adaptations on ultrasound may precede pain or symptoms and thereby represent the first pathologic changes in the pitching elbow. ${ }^{14-16}$ Therefore, it is vital to understand how medial elbow stressors contribute to these changes to prevent injuries.

Recent findings support the accuracy and accessibility of kinematic parameters such as medial elbow torque with wearable sensor technology. It remains unknown what association these metrics have on the adaptive changes of the medial elbow, which have been implicated with increased pitcher injury. In addition, understanding the relationship between kinematic parameters and anatomic adaptive changes in the elbow may help identify strategies toward injury prevention in these athletes. The purpose of this study was to determine whether elbow torque was associated with anatomic adaptations of the medial elbow following a season of competitive pitching. The authors hypothesized that pitchers who impart greater medial elbow stresses during their throwing motion would develop increased changes in UCL thickness and elbow laxity following a season of competitive pitching.

\section{Methods}

This is a prospective, observational study that sought to evaluate whether elbow torque measured during the pitching motion is a predictive factor for sonographic anatomical adaptations in the pitching elbow after a season of competitive pitching. The investigators previously published their findings in this cohort of pitchers during preseason testing. ${ }^{21}$ This follow-up study compared postseason testing with preseason testing in the cohort of pitchers who were available for follow-up. Pitchers who left their college campus to return home for the summer break, or who graduated, were unavailable to follow-up for postseason testing. This study was conducted with approval from the institutional review board (No. 12481) at Henry Ford Hospital. Each participant gave informed consent before data collection. This study was conducted from January 2019 to June 2019. Pitchers from 3 National Collegiate Athletic Association Division II college baseball teams located in a metropolitan area in the Midwest of the United States were recruited for this study and underwent a standardized informed consent process with documentation. All active pitchers were eligible for consideration to the study. Players older than 18 who described their primary position as pitcher were included. Exclusion criteria included age $<18$ years, if they were injured, recovering from injury, or otherwise not currently pitching at full effort, reported a history of surgery on the dominant extremity, predominately pitch side arm or "submarine" style (as elbow torque measurements may be inflated), ${ }^{22,23}$ and inability to attend required data collection sessions or follow-up postseason.

Player data were collected at team practice sessions by 2 orthopaedic surgery residents (T.R.J. and L.S.K.). Data included demographic characteristics via an intake form and pitching kinematic measurements. Demographic measurements included height, weight, body mass index, total arm length, and elbow circumference. The total arm length was considered the distance from the lateral aspect of the acromion to the distal aspect of the fifth digit. Elbow circumference was measured around the medial and lateral epicondyles of the humerus. Anthropometric and arm length measurements in this study were consistent with previous studies implementing this wearable device. ${ }^{5,6,24,25}$

Pitchers were fitted with an appropriately size athletic sleeve containing a medial elbow sensor, which recorded accelerometer and gyroscope data to a mobile phone application (motusTHROW $\mathrm{v}$ 8.3.3; Motus Global, Seattle, WA). The device manufacturer and vendor had no involvement in funding or design of this study. The recorded data are indirectly measured by referencing the position of the arm relative to the ground and include elbow torque (Newton meters, $\mathrm{Nm}$ ), arm slot (degrees, ${ }^{\circ}$ ), arm speed (rotations per minute, rpm), and shoulder rotation $\left({ }^{\circ}\right)$. Arm slot is calculated by the angle of the forearm relative to the ground at the time of ball release, with lower angles correlating to lower point of releasing the ball. Arm rotation is the maximum angle of the forearm relative to the ground during the transition from late cocking to 


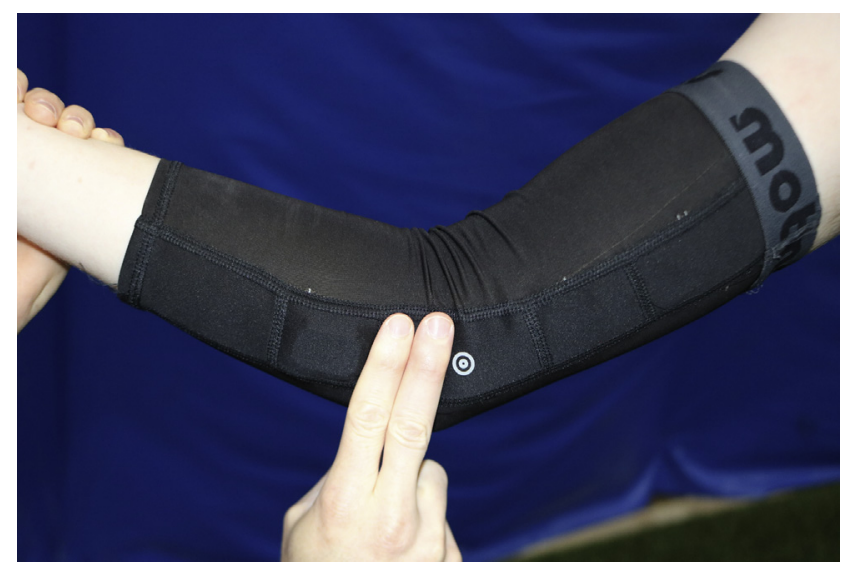

Fig 1. Wearable baseball compression sleeve with a sensor device. Pictured is a wearable baseball compression sleeve on a right elbow, with a sensor device at the medial elbow. The device is placed so that the sensor rests 1.5 inches (approximately 2 fingerbreadths) distal to the medial epicondyle of the humerus, as directed by the device manufacturer's instructions. The medial epicondyle is depicted by the circle on the sleeve.

early acceleration, serving as a marker of shoulder external rotation. The device was placed so that the sensor rested 1.5 inches distal to the medial epicondyle of the humerus, as directed by the device manufacturer's instructions (Fig 1). This device has been shown to have significant correlation with video motion capture and has been studied extensively. ${ }^{4-6,8,26-28}$

Consistent with previous literature, preseason data were collected within 2 weeks of the first game of the season and postseason data were collected within 1 week of the final game. ${ }^{18,25}$ For each session, testing while using the wearable sensor technology compression sleeve followed a similar format to prior studies. 5, 6,23,28,29 Participants were allowed as much time as they required to warm up using their typical routine. Players were then instructed to emulate livegame pitching. They were given as many opportunities as they needed to throw while wearing the baseball sensor to become accustom to it. When they were ready, the pitchers were recorded throwing 5 consecutive fastball pitches at maximum effort. All pitches were thrown from the mound at a standard distance of 60 feet and 6 inches (18.4 meters). Pitches were considered erroneous and not counted toward data collection if the ball could not be reasonably stopped by the catcher. Furthermore, ball velocity was measured in miles per hour using a radar gun situated behind the player (Stalker Sport 2 radar gun; Stalker Radar, Richardson, TX). Data output by the device was collected from the mobile phone application and recorded for analysis.

Pitchers underwent formal dynamic ultrasound imaging of the dominant elbow in both the preseason and postseason, which took place within 1 week of their pitching session. Measurements were taken on a day where they had not participated in any baseball-related activities to avoid capturing transient changes in joint laxity. ${ }^{30}$ Ultrasound imaging was performed in the radiology suite of the main institution by a single experienced musculoskeletal sonographer (Logiq E9; GE Healthcare, Little Chalfont, UK). In accordance with the American Institute of Ultrasound in Medicine guidelines, all images were taken in both a long- and short-axis plane relative to structure being imaged. To evaluate the anterior band of the UCL, the athletes were seated with their shoulder maximally externally rotated and arm place in $30^{\circ}$ of flexion as measured with a goniometer. Examination of the UCL dynamically and the elbow medial joint line (UHJS) was achieved by placing pitchers' elbow in $30^{\circ}$ of flexion while a single examiner applied a valgus force at the ulnar border of the forearm while the upper arm was stabilized. A standardized 100lb valgus force was applied using a handheld dynamometer (Manual Muscle Testing System Model 01165; Lafayette, Lafayette, IN). Dynamic imaging of the UHJS was captured by the sonographer throughout the entire application of the valgus load.

All ultrasound images were deidentified and evaluated by 2 blinded, fellowship-trained, musculoskeletal radiologists. Assessment of the UCL thickness, UHJS with (elbow laxity) and without valgus load, and presence of loose bodies was conducted in identical fashion to prior studies. ${ }^{18,19,25}$ The Modified Jacobson-Ward technique was used to measure the UCL. ${ }^{31}$ Parallel cortices were used for measuring UHJS, with particular attention to omitting bone spurs.

\section{Statistical Analysis}

A power analysis was performed based off recent pitching studies using the same wearable sensor technology. $5,6,27,28$ With a power of $80 \%$ (beta level of 0.80 , alpha level of 0.05), a sample size of 14 players per group was found, for a total of 28 players, to detect a significant difference of $2.5 \mathrm{Nm}$ in elbow torque between pitchers with and without elbow laxity. The preseason analysis, published separately, demonstrated greater than $2.5 \mathrm{Nm}$ difference in elbow torque among pitchers with loaded UHJS $\geq 4.4 \mathrm{~mm}$ (43.9 vs 48.6 , $P<.001)$ and delta UHJS $\geq 1.25 \mathrm{~mm}$ (44.2 vs 47.7, $P<.001) .{ }^{21}$ The present investigation was a postseason assessment of the same cohort of pitchers, compared with their preseason values; as such, the sample size was determined by those pitchers available for followup.

Statistical analysis was performed by a trained statistician. All data is described using appropriate statistical analysis. Continuous variables reported include means, medians, minimums, 25 th percentile, 75 th percentile, 
Table 1. Comparison of Player Demographic and Arm Length Measurements Between Seasons

\begin{tabular}{|c|c|c|c|}
\hline & Preseason Only $(\mathrm{N}=9)$ & Pre- and Postseason $(\mathrm{N}=19)$ & $P$ Value \\
\hline$\overline{\text { Age, } y}$ & $20.7 \pm 1.4$ & $19.9 \pm 1.2$ & .14 \\
\hline Right-hand dominance & $100 \%$ & $63.2 \%$ & .06 \\
\hline College year & & & .10 \\
\hline Sophomores & $2(22.2 \%)$ & $6(31.6 \%)$ & \\
\hline Juniors & $4(44.4 \%)$ & $4(21.1 \%)$ & \\
\hline Seniors & $3(33.3 \%)$ & $2(10.5 \%)$ & \\
\hline Weight, kg & $89.8 \pm 8.6$ & $79.9 \pm 12.1$ & .04 \\
\hline BMI & $25.2 \pm 2.6$ & $23.3 \pm 3.4$ & .15 \\
\hline Pitcher position & & & .85 \\
\hline Starter & $5(55.6 \%)$ & $8(42.1 \%)$ & \\
\hline Reliever & $3(33.3 \%)$ & $9(47.4 \%)$ & \\
\hline Closer & $1(11.1 \%)$ & $2(10.5)$ & \\
\hline Large & $0(0 \%)$ & $1(5.26 \%)$ & \\
\hline Arm length, $\mathrm{cm}$ & $78.4 \pm 3.9$ & $77.5 \pm 4.9$ & .61 \\
\hline Elbow circumference, $\mathrm{cm}$ & $28.5 \pm 1.7$ & $27.5 \pm 1.8$ & .17 \\
\hline
\end{tabular}

NOTE. Continuous variables are presented using mean \pm standard deviation, and categorical variables are presented as number (percentage). Statistically significant $P$ values are indicated in bold $(P<.05)$.

BMI, body mass index; NCAA, National Collegiate Athletic Association.

maximum, and standard deviation for nonrepeated continuous variables, whereas repeated continuous variables were expressed as the least-squared means and standard errors. Counts and percentages were reported for categorical variables. Metrics captured by repeated measurement were described using adjusted means and standard errors. All player demographics are compared between groups using chi-square or Fisher's exact tests for categorical variables and using analysis of variance or Wilcoxon rank-sum tests. To account for pitching metrics being collected 5 times on each player, a univariate repeated-measures analysis of variance using a generalized estimating equations approach was used and results are given as least-squared means (beta estimate) with standard errors. To demonstrate the level of agreement between raters, intraclass correlations were obtained for continuous variables. Intraclass correlation coefficients $(2,1)$ is reported since all subjects are rated by the same raters who are assumed to be a random subset of all possible raters. Kappa statistics are calculated for categorical variables using $2 \times 2$ tables. Statistical significance is set at $P<.05$. All analyses are performed using SAS, 9.4 (SAS Institute Inc, Cary, NC).

\section{Results}

\section{Demographic Characteristics}

Twenty-eight collegiate pitchers underwent preseason sonographic and kinematic testing. Nineteen pitchers were available for postseason testing. Demographics and anthropometric features of eligible pitchers are outlined in Table 1. When we compared pitchers who completed both pre- and postseason sessions with those who were only present in the preseason, pitchers who presented to the postseason session had, on average, fewer years of experience playing baseball $(14.7 \pm 1.5$ years vs $16.4 \pm 1.9$ years, $P=.02)$ and were lighter $(79.9 \pm 12.1 \mathrm{~kg}$ vs $89.8 \pm 8.6$ $\mathrm{kg}, P=.04)$. There were no other statistically significant differences.

\section{Pitching Kinematic and Dynamic Ultrasound Measurements}

Table 2 demonstrates pitching and ultrasound measurements comparing preseason and postseason data collection periods. Regarding kinematic measurements, when compared with preseason values, there was a significant increase in postseason ball velocity $(77.1 \pm$ 0.4 vs $75.8 \pm 0.4, P=.04)$. There was no significant change in noted in arm slot, arm speed, arm rotation, or elbow torque following a season of pitching.

When evaluating adaptive anatomic changes to the medial elbow, players had a significant increase in UCL thickness $(1.92 \pm 0.09$ vs $1.56 \pm 0.09 \mathrm{~mm}, P<.01)$ and increase in the loaded UHJS measurements (4.61 \pm 0.21 vs $4.09 \pm 0.20 \mathrm{~mm}, P=.063$ ) following a season of pitching. There was a significant increase in elbow laxity, as measured by delta UHJS (UHJS widening from an unloaded to loaded state), in the postseason 
Table 2. Preseason Versus Postseason Changes in Pitching Kinematic and Dynamic Ultrasound Measurements

\begin{tabular}{|c|c|c|c|}
\hline & Preseason & Postseason & $P$ Value \\
\hline Ball velocity, mph & $75.8 \pm 0.4$ & $77.1 \pm 0.4$ & .041 \\
\hline Arm slot, ${ }^{\circ}$ & $45.7 \pm 1.6$ & $43.0 \pm 1.6$ & .256 \\
\hline Arm speed, rpm & $888.2 \pm 8.4$ & $892.2 \pm 8.4$ & .744 \\
\hline Arm rotation, ${ }^{\circ}$ & $149.5 \pm 1.5$ & $152.4 \pm 1.5$ & .199 \\
\hline Elbow torque, $\mathrm{Nm}$ & $46.1 \pm 0.9$ & $45.7 \pm 0.9$ & .72 \\
\hline UCL thickness, mm & $1.56 \pm 0.09$ & $1.92 \pm 0.09$ & .001 \\
\hline Unloaded UHJS, mm & $2.94 \pm 0.10$ & $2.85 \pm 0.10$ & .364 \\
\hline Loaded UHJS & $4.09 \pm 0.20$ & $4.61 \pm 0.21$ & .063 \\
\hline UHJS delta & $1.15 \pm 0.22$ & $1.77 \pm 0.23$ & .028 \\
\hline
\end{tabular}

NOTE. Continuous variables are presented using mean \pm standard deviation, and categorical variables are presented as number (percentage). Statistically significant $P$ values are indicated in bold $(P<$ $.05)$.

mph, miles per hour; rpm, rotations per minute; UCL, ulnar collateral ligament; UHJS, ulnohumeral joint space.

compared with the preseason $(1.77 \pm 0.23$ vs $1.15 \pm$ $0.22 \mathrm{~mm}, P=.028)$.

\section{Pitching Kinematics: Association With Ultrasound Findings}

Table 3 represents the association between preseason pitching measurements and postseason ultrasound findings. Preseason arm slot demonstrated significant association with change in UCL thickness $(-0.03 \pm 0.01$ beta estimate, $P=.011$ ), indicating that for each degree of lower arm slot (later ball release), UCL thickness increased by $0.03 \mathrm{~mm}$ after the season. Preseason elbow torque demonstrated significant association with both postseason loaded UHJS and change in loaded UHJS $(-0.03 \pm 0.01$ beta estimate, $P=.04)$, indicating that for every $1-\mathrm{Nm}$ increase in elbow torque, elbow laxity decreased by $0.03 \mathrm{~mm}$ after the season. No other preseason pitching kinematic variable was associated with loaded UHJS at any given time point.

\section{Demographic Factors: Associations With Elbow Torque}

Table 4 demonstrates the association of pitcher demographic characteristics with elbow torque measurements obtained by wearable sensor technology. Postseason elbow torque demonstrated significant inverse associations (beta estimate [standard error]) with player weight $(-0.45[0.18], P=.027)$ and total dominant arm length $(-1.34$ [0.42], $P=.006)$, such that each additional kilogram of body weight or each additional centimeter of arm length was associated with a 0.45 or 1.34 Newton-meter reduction of elbow torque, respectively. The change in elbow torque over the course of a season was noted to inversely relate to player age $(-3.60$ [1.68], $P=.046)$, such that an age increase of one year was associated with a 3.60 Newton-meter reduction in elbow torque after the season.

\section{Discussion}

Preseason pitching kinematic measurements were significantly associated with postseason adaptations present on ultrasound, with reduced arm slot (lower ball release) correlating to increased UCL thickness and increased elbow torque correlating to reduced elbow laxity, after a season of pitching. However, the magnitude of these findings is small in value and the clinical significance of these changes remains unknown. Age, weight, and arm length were found to be protective of medial elbow torque. In addition, the adaptations present on ultrasound in this study were consistent with prior literature, including increased UCL thickness and elbow laxity following the season.

In a single time-point preseason analysis of this cohort of 28 collegiate pitchers, Khalil et al. ${ }^{21}$ investigated the relationship between elbow torque and sonographic adaptations to the medial elbow. The authors found a significant relationship between greater medial elbow torque during pitching and a thicker UCL and stiffer elbow (reduced elbow laxity) on dynamic ultrasound of pitchers. The authors noted that these findings were a snapshot of a single time-point in the preseason among asymptomatic pitchers. The 19 pitchers who were available for examination after a season of competitive pitching were examined in this present study, further corroborating these relationships. Pitchers with increased preseason elbow torque demonstrated reduced postseason elbow laxity. In addition, pitchers with decreased preseason arm slot demonstrated significantly greater UCL thickness after a season of pitching. Reduced arm slot (i.e., lower ball release) has shown to generate increased "whip" at ball release and subsequently greater elbow stresses. ${ }^{6,8}$ These findings taken together indicate that pitchers with greater elbow torque in their throwing motion, and potentially a lower ball release, demonstrate greater adaptations on ultrasound, including a thicker UCL and reduced elbow laxity after a season of pitching. A possible explanation is that pitchers with greater demands (i.e., elbow torque, "whip" at ball release) placed on their medial elbow initially develop protective mechanisms, such as greater static and dynamic restraints of the elbow with a thicker UCL and reduced elbow laxity, which this cohort of asymptomatic pitchers may represent. However, continuous overhead activity and repetition may lead to chronic attenuation of the medial elbow, progressive elbow laxity in later stages, and subsequent increased risk of injury in symptomatic pitchers. ${ }^{32,33}$

Previous literature has suggested that increased elbow stresses in overhead throwing athletes may lead to ulnohumeral adaptive changes on ultrasound, as demonstrated in this study, such as UCL thickening. ${ }^{14,18,25,30}$ In a cross-sectional study of 102 pitchers, Atanda et al. ${ }^{14}$ evaluated pitching volume in 
Table 3. Effect of Pitching Kinematic Variables on Dynamic Ultrasound Measurements

\begin{tabular}{|c|c|c|c|c|c|c|c|c|}
\hline \multirow[b]{2}{*}{ Preseason Pitching Variable } & \multicolumn{2}{|c|}{ Postseason UCL Thickness } & \multicolumn{2}{|c|}{ Change in UCL Thickness } & \multicolumn{2}{|c|}{ Postseason UHJS Loaded } & \multicolumn{2}{|c|}{ Change in UHJS Loaded } \\
\hline & Est (SE) & $P$ Value & Est (SE) & $P$ Value & Est (SE) & $P$ Value & Est (SE) & $P$ Value \\
\hline Elbow torque & $0.005(0.01)$ & .606 & $0.0004(0.01)$ & .965 & $-0.03(0.01)$ & .042 & $-0.03(0.01)$ & .045 \\
\hline Arm slot & $-0.016(0.01)$ & .156 & $-0.03(0.01)$ & .011 & $0.004(0.03)$ & .912 & $0.01(0.03)$ & .667 \\
\hline Arm rotation & $-0.01(0.01)$ & .371 & $-0.001(0.01)$ & .991 & $-0.02(0.03)$ & .523 & $-0.03(0.03)$ & .283 \\
\hline Ball velocity & $-0.012(0.04)$ & .745 & $-0.008(0.03)$ & .787 & $0.09(0.10)$ & .368 & $0.13(0.10)$ & .218 \\
\hline
\end{tabular}

NOTE. Univariate relationships for continuous variables are presented as beta estimate (standard error). Interpretation for beta estimates is as follows: for every l-unit increase in a pitching kinematic measurement, the ultrasound measurement changes by the magnitude of the beta estimate (i.e., for every 1-degree reduction in preseason arm slot, the change in UCL thickness from preseason to postseason in the cohort of players increased by $0.03 \mathrm{~mm}(0.01)$ significantly).

Statistically significant $P$ values are indicated in bold $(P<.05)$.

UCL, ulnar collateral ligament; UHJS, ulnohumeral joint space.

adolescent pitchers and found that the UCL was significantly thicker in pitchers who threw more than 67 pitches per appearance $(4.69 \mathrm{~mm}$ vs $4.14 \mathrm{~mm}, P=$ $.03)$. In a prospective observational study of 22 high school pitchers, Keller et al. ${ }^{18}$ found significant increases in UCL thickness and UCL substance heterogeneity $(P=.02$ and $P=.001)$ after a season of pitching relative to preseason measurements. The present study aligns with the aforementioned literature, demonstrating that UCL thickness increases after a season of pitching. In addition, the present findings suggest that kinematic parameters of the throwing motion measured by wearable sensor technology are associated with the sonographic adaptations observed in the literature. Specifically, a lower arm slot, defined by a later ball release, was related to increased UCL thickness in pitchers after the season. These findings are consistent with the concept that a lower arm slot generates greater "whip" at the medial elbow, as demonstrated by Okoroha et al. ${ }^{6}$ and Camp et al., ${ }^{8}$ who found greater elbow stresses in pitchers with lower arm slot. Extrapolating those findings would explain why pitchers in the present cohort with lower arm slot may develop greater UCL thickness.
Studies investigating the future development of pain and injury have provided insight into the clinical significance of sonographic findings of the medial elbow. Studies conducting serial ultrasound imaging have demonstrated that UCL thickness and UHJS widening progress after a season of pitching. ${ }^{16,18,25,32}$ Tajika et al. ${ }^{33}$ evaluated 122 high school baseball pitchers during the preseason with ultrasound of their dominant elbow and found significantly greater UCL thickness and UHJS widening in pitchers with a history of elbow pain. Furthermore, Shanley et al. ${ }^{32}$ performed dynamic ultrasounds on 70 asymptomatic professional baseball pitchers, following these pitchers prospectively, and discovered that the 7 pitchers who subsequently sustained a UCL injury had significantly greater elbow laxity than the 63 who remained healthy $(6.5 \pm 1.2 \mathrm{vs}$ $5.3 \pm 1.2 \mathrm{~mm}, P=.01$ ). The findings of the current study corroborate that collegiate pitchers demonstrate adaptations to UCL thickness and elbow laxity after a season of competitive pitching. Counterintuitively, pitchers in the present study with greater medial elbow torque in the preseason were associated with reduced elbow laxity in the postseason. This highlights the spectrum of anatomic adaptations that overhead

Table 4. Association Between Player Demographic Characteristics and Elbow Torque

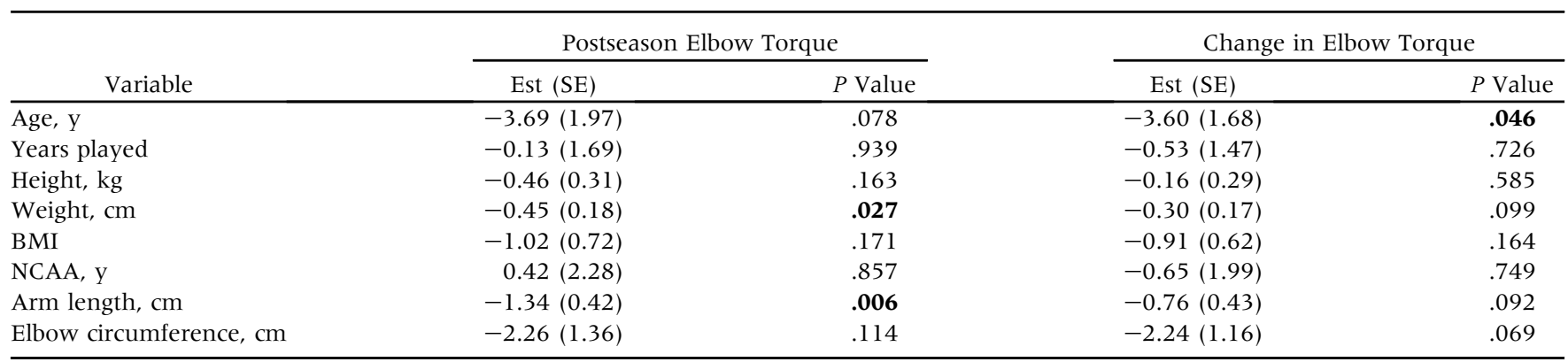

NOTE. Univariate relationships for continuous variables are presented as beta estimate (standard error). Interpretation for beta estimates is as follows: for every l-unit increase in a demographic measurement, the wearable sensor measurement changes by the magnitude of the beta estimate (i.e., for every 1-year increase in player age, the change in elbow torque from preseason to postseason in the cohort of players decreased by 3.60 (1.68) Newton-meter significantly). Statistically significant $P$ values are indicated in bold $(P<.05)$.

BMI, body mass index; NCAA, National Collegiate Athletic Association. 
throwers may develop, warranting further prospective studies to delineate which adaptations are normal responses to stressors versus pathologic adaptations at risk of injury. Additional work must be done to elucidate the role of wearable sensors in monitoring pitching mechanics and the implications of monitoring on injury prevention as related to pathoanatomic adaptations present on advanced imaging.

Several studies have sought to evaluate the role of anthropomorphic characteristics in predicting medial elbow torque recorded by wearable sensor technology while pitching. ${ }^{5,6,23,29}$ In a cohort study, Lizzio et al. ${ }^{29}$ evaluated elbow torque in 18 professional baseball pitchers and found that an increase in body mass index was independently associated with a reduction in elbow torque $(P=.035)$. Likewise, Makhni et al. ${ }^{5}$ performed a cohort study of 37 collegiate pitchers and demonstrated that larger elbow circumference was a predictor for reduced medial elbow torque $(P=.021)$. Okoroha et al. ${ }^{6}$ evaluated predictors for elbow torque in 20 youth baseball players and found that increased age and increased arm length were both independently associated with reductions in elbow torque $(P<.001)$. Similar to previous studies, the present investigation also found an increase in age, body weight, and arm length was related to reduced elbow torque $(P=.046$, $P=.027$, and $P=.006$, respectively). Increased body mass and elbow circumference are thought to portend a protective effect by increasing dynamic stabilization of the medial elbow via greater load distribution. Increased arm length is thought to portend a mechanical advantage by providing a larger moment arm in which pitchers can produce increase speeds with lower medial elbow stresses, and the influence of age is thought to be correlated with athletic experience, as older players develop more consistent and efficient pitching kinematics. $^{22,34}$

\section{Limitations}

This investigation presents with several limitations. As athletes from multiple collegiate universities were recruited, players' exposure to training regimens, playing time, practice protocols, and previous workload volume was unable to be controlled for when performing the statistical analysis. While athletes were all tested at equivalent time points, it was not possible to meet all players for multiple sessions and serial measurements, therefore no interrater reliability analysis was possible. In addition, the small sample size is a result of all available athletes present for recruitment and subsequent follow-up. The loss of follow-up of 9 athletes was due to their lack of availability since many athletes returned to their out-of-state homes for summer vacation immediately following the conclusion of playoffs. While this may affect the data, only weight and years of experience were significantly different between pitchers who did and did not follow-up in the postseason, likely as a result of older pitchers graduating college. With regards to pitching kinematic measurements, these calculations by the wearable sensor is determined by a gyroscope embedded in an elbow sleeve, which derives these values based on the sensor's position on the elbow relative to the ground. This mean that it is not possible to account for any contributions of shoulder girdle, trunk, or pelvic motion to these values as would be performed by a 3-dimensional motion video analysis. With regard to the ultrasound measurements, pitchers were instructed to relax during dynamic elbow laxity examination, which likely does not account for the contributions of dynamic stabilizers to the elbow (i.e., flexor pronator mass) that are typically activated during the pitching motion. Due to the novel nature of this study, when interpreting correlational results between pitching kinematics and ultrasound changes, previous literature was used to extrapolate the clinical relevance. Ultimately it should be reiterated that the present investigation represents a snapshot of healthy, asymptomatic athletes' measurements at 2 time points, and our findings may not be representative of symptomatic players nor might these findings be representative of youth and adolescent patient populations.

\section{Conclusions}

After a season of competitive pitching, adaptive changes of the medial elbow were demonstrated on dynamic ultrasound. However, the influence of pitching kinematic measurements on these adaptations are of small magnitude and unknown clinical significance. Although wearable sensor technology may have value in trending individual pitcher kinematics, no discrete threshold appears to predict the development of adaptive changes at the elbow.

\section{Acknowledgments}

The authors acknowledge Anastasia M. Khalil for her assistance in recruiting, consenting, and organizing subject participation.

\section{References}

1. Dun S, Kingsley D, Fleisig GS, Loftice J, Andrews JR. Biomechanical comparison of the fastball from wind-up and the fastball from stretch in professional baseball pitchers. Am J Sports Med 2008;36:137-141.

2. Escamilla RF, Barrentine SW, Fleisig GS, et al. Pitching biomechanics as a pitcher approaches muscular fatigue 
during a simulated baseball game. Am J Sports Med 2007;35:23-33.

3. Fleisig GS, Andrews JR. Prevention of elbow injuries in youth baseball pitchers. Sports Health 2012;4:419-424.

4. Boddy KJ, Marsh JA, Caravan A, Lindley KE, Scheffey JO, $\mathrm{O}^{\prime}$ Connell ME. Exploring wearable sensors as an alternative to marker-based motion capture in the pitching delivery. PeerJ 2019;7:e6365.

5. Makhni EC, Lizzio VA, Meta F, Stephens JP, Okoroha KR, Moutzouros V. Assessment of elbow torque and other parameters during the pitching motion: Comparison of fastball, curveball, and change-up. Arthroscopy 2018;34: 816-822.

6. Okoroha KR, Lizzio VA, Meta F, Ahmad CS, Moutzouros V, Makhni EC. Predictors of elbow torque among youth and adolescent baseball pitchers. Am J Sports Med 2018;46:2148-2153.

7. Okoroha KR, Meta F, Meldau J, et al. Predictors of elbow torque in youth and adolescent baseball pitchers using a new mobile sensor: fastballs versus breaking pitches. Arthroscopy 2018;34:e24.

8. Camp CL, Tubbs TG, Fleisig GS, et al. The relationship of throwing arm mechanics and elbow varus torque: Within-subject variation for professional baseball pitchers across 82,000 throws. Am J Sports Med 2017;45: 3030-3035.

9. Anz AW, Bushnell BD, Griffin LP, Noonan TJ, Torry MR, Hawkins RJ. Correlation of torque and elbow injury in professional baseball pitchers. Am J Sports Med 2010;38: 1368-1374.

10. Gregory B, Nyland J. Medial elbow injury in young throwing athletes. Muscles Ligaments Tendons J 2013;3: 91-100.

11. Rossy WH, Oh LS. Pitcher's elbow: Medial elbow pain in the overhead-throwing athlete. Curr Rev Musculoskelet Med 2016;9:207-214.

12. DeFroda SF, Goodman AD, Gil JA, Owens BD. Epidemiology of elbow ulnar collateral ligament injuries among baseball players: National Collegiate Athletic Association Injury Surveillance Program, 2009-2010 through 20132014. Am J Sports Med 2018;46:2142-2147.

13. Chalmers PN, Wimmer MA, Verma NN, et al. The relationship between pitching mechanics and injury: A review of current concepts. Sports Health 2017;9:216-221.

14. Atanda A Jr, Averill LW, Wallace M, Niiler TA, Nazarian LN, Ciccotti MG. Factors related to increased ulnar collateral ligament thickness on stress sonography of the elbow in asymptomatic youth and adolescent baseball pitchers. Am J Sports Med 2016;44:3179-3187.

15. Atanda A Jr, Buckley PS, Hammoud S, Cohen SB, Nazarian LN, Ciccotti MG. Early anatomic changes of the ulnar collateral ligament identified by stress ultrasound of the elbow in young professional baseball pitchers. Am J Sports Med 2015;43:2943-2949.

16. Ciccotti MG, Atanda A Jr, Nazarian LN, Dodson CC, Holmes L, Cohen SB. Stress sonography of the ulnar collateral ligament of the elbow in professional baseball pitchers: A 10-year study. Am J Sports Med 2014;42: 544-551.
17. Erb J, Sherman H, Williard S, Bui J, Kachingwe A. Ultrasound study of elbow ulnar collateral ligament changes in collegiate baseball players: A pilot study. J Bodyw Mov Ther 2017;21:259-266.

18. Keller RA, Marshall NE, Bey MJ, et al. Pre- and postseason dynamic ultrasound evaluation of the pitching elbow. Arthroscopy 2015;31:1708-1715.

19. Marshall NE, Keller RA, Okoroha K, et al. Radiostereometric evaluation of tendon elongation after distal biceps repair. Orthop J Sports Med 2016:4:2325967116672620.

20. Marshall NE, Keller RA, Van Holsbeeck M, Moutzouros V. Ulnar collateral ligament and elbow adaptations in high school baseball pitchers. Sports Health 2015;7:484-488.

21. Khalil LS, Meta FS, Tramer JS, et al. Elbow torque is reduced in asymptomatic college pitchers with elbow laxity: A dynamic ultrasound study. Arthroscopy 2021;37: 852-861.

22. Aguinaldo AL, Chambers H. Correlation of throwing mechanics with elbow valgus load in adult baseball pitchers. Am J Sports Med 2009;37:2043-2048.

23. Smith DG, Swantek AJ, Gulledge CM, et al. Relationship between glenohumeral internal rotation deficit and medial elbow torque in high school baseball pitchers. Am J Sports Med 2019;47:2821-2826.

24. Downs JL, Wasserberger KW, Barfield JW, et al. Increased upper arm length and loading rate identified as potential risk factors for injury in youth baseball pitchers. Am J Sports Med 2021;49:3088-3093.

25. Khalil LS, Okoroha KR, Jildeh TR, et al. Do anatomic changes found in the throwing arm after a season of pitching resolve with off-season rest? A dynamic ultrasound study. JSES Open Access 2019;3:338-343.

26. Khalil LS, Jildeh TR, Taylor KA, et al. The relationship between shoulder range of motion and elbow stress in college pitchers. J Shoulder Elbow Surg 2021;30:504-51 1.

27. Okoroha KR, Meldau JE, Jildeh TR, Stephens JP, Moutzouros V, Makhni EC. Impact of ball weight on medial elbow torque in youth baseball pitchers. J Shoulder Elbow Surg 2019;28:1484-1489.

28. Okoroha KR, Meldau JE, Lizzio VA, et al. Effect of fatigue on medial elbow torque in baseball pitchers: A simulated game analysis. Am J Sports Med 2018;46:2509-2513.

29. Lizzio VA, Gulledge CM, Smith DG, et al. Predictors of elbow torque among professional baseball pitchers. J Shoulder Elbow Surg 2020;29:316-320.

30. Hattori H, Akasaka K, Otsudo T, Hall T, Amemiya K, Mori Y. The effect of repetitive baseball pitching on medial elbow joint space gapping associated with 2 elbow valgus stressors in high school baseball players. J Shoulder Elbow Surg 2018;27:592-598.

31. Shukla M, Keller R, Marshall N, et al. Ultrasound evaluation of the ulnar collateral ligament of the elbow: Which method is most reproducible? Skelet Radiol 2017;46:4.

32. Shanley E, Smith M, Mayer BK, et al. Using stress ultrasonography to understand the risk of UCL injury among professional baseball pitchers based on ligament morphology and dynamic abnormalities. Orthop J Sports Med 2018;6:2325967118788847. 
33. Tajika T, Yamamoto A, Oya N, et al. The morphologic change of the ulnar collateral ligament of elbow in high school baseball pitchers, with and without symptoms, by sonography. J Shoulder Elbow Surg 2016;25:1223-1228.
34. Stodden DF, Fleisig GS, McLean SP, Andrews JR. Relationship of biomechanical factors to baseball pitching velocity: Within pitcher variation. J Appl Biomech 2005;21: 44-56. 